\title{
Evolution of MS lesions to black holes under DNA vaccine treatment
}

\author{
Athina Papadopoulou • Stefanie von Felten $\cdot$ Stefan Traud $\cdot$ Amena Rahman • \\ Joanne Quan - Robert King • Hideki Garren - Lawrence Steinman • \\ Gary Cutter $\cdot$ Ludwig Kappos $\cdot$ Ernst Wilhelm Radue
}

Received: 27 September 2011/Revised: 6 December 2011/Accepted: 8 December 2011/Published online: 6 January 2012

(c) Springer-Verlag 2011

\begin{abstract}
Persistent black holes (PBH) are associated with axonal loss and disability progression in multiple sclerosis (MS). The objective of this work was to determine if BHT-3009, a DNA plasmid-encoding myelin basic protein (MBP), reduces the risk of new lesions becoming PBH, compared to placebo, and to test if pre-treatment serum anti-MBP antibody levels impact on the effect of BHT3009 treatment. In this retrospective, blinded MRI study,
\end{abstract}

Part of the results of this study was presented as a poster-presentation at the 25th Congress of the European committee for treatment and research in multiple sclerosis (ECTRIMS) in Düsseldorf, Germany, in September 2009. Session: immunomodulation-2, P820: "Conversion of new inflammatory lesions to persistent black holes in patients with relapsing multiple sclerosis participating in a phase II trial of DNA vaccine encoding myelin basic protein (BHT-3009)".

Electronic supplementary material The online version of this article (doi:10.1007/s00415-011-6361-x) contains supplementary material, which is available to authorized users.

A. Papadopoulou · L. Kappos $(\bowtie)$

Neurology Clinic, Department of Neurology, University Hospital Basel, Petersgraben 4, 4031 Basel, Switzerland

e-mail: 1kappos@uhbs.ch

A. Papadopoulou

e-mail: papadopouloua@uhbs.ch

S. von Felten

Clinical Trial Unit, University Hospital Basel, Basel,

Switzerland

e-mail: vonfeltens@uhbs.ch

S. Traud · E. W. Radue

Medical Image Analysis Center (MIAC),

University Hospital Basel, Basel, Switzerland

e-mail: stefan.traud@miac.ch

E. W. Radue

e-mail: eradue@uhbs.ch we reviewed MRI scans of 155 MS patients from a doubleblind, randomized, phase II trial with three treatment arms (placebo, 0.5 and $1.5 \mathrm{mg}$ BHT-3009). New lesions at weeks 8 and 16 were tracked at week 48 and those appearing as T1-hypointense were classified as PBH. A subset of 46 patients with available pre-treatment serum anti-MBP IgM levels were analyzed separately. Overall, there was no impact of treatment on the risk for PBH. However, there was a significant interaction between antiMBP antibodies and treatment effect: patients receiving $0.5 \mathrm{mg}$ BHT-3009 showed a reduced risk of $\mathrm{PBH}$ with higher antibody levels compared to placebo $(p<0.01)$. Although we found no overall reduction of the risk for $\mathrm{PBH}$ in treated patients, there may be an effect of low-dose BHT-3009, depending on the patients' pre-treatment immune responses.

\footnotetext{
A. Rahman · J. Quan $\cdot$ R. King $\cdot$ H. Garren

Bayhill Therapeutics, Palo Alto, CA, USA

e-mail: arahman31@gmail.com

J. Quan

e-mail: jquan@bayhilltx.com

R. King

e-mail: rking@bayhilltx.com

H. Garren

e-mail: hgarren@bayhilltx.com

L. Steinman

Stanford University, Stanford, USA

e-mail: steinman@stanford.edu

G. Cutter

Department of Biostatistics, University of Alabama,

Birmingham, USA

e-mail: cutterg@prodigy.net
} 
Keywords Multiple sclerosis - BHT-3009 .

DNA vaccine $\cdot$ Persistent black holes

$\begin{array}{ll}\text { Abbreviations } \\ \text { ABH } & \text { Acute black holes } \\ \text { CL } & \text { Candidate lesions } \\ \text { CSF } & \text { Cerebrospinal fluid } \\ \text { EDSS } & \text { Expanded Disability Status Scale } \\ \text { Gd } & \text { Gadolinium } \\ \text { MBP } & \text { Myelin basic protein } \\ \text { MS } & \text { Multiple sclerosis } \\ \text { MSSS } & \text { MS Severity Score } \\ \text { MTR } & \text { Magnetization transfer ratio } \\ \text { NAA } & \text { N-Acetyl aspartate } \\ \text { NAGM } & \text { Normal appearing grey matter } \\ \text { NAWM } & \text { Normal appearing white matter } \\ \text { NUM } & \text { Number of human MBP peptide epitopes } \\ & \text { recognized by serum antibodies } \\ \text { PBH } & \text { Persistent black holes } \\ \text { PD-w SE } & \text { Proton density-weighted sequence } \\ \text { RRMS } & \text { Relapsing remitting multiple Sclerosis } \\ \text { T1-w SE } & \text { T1-weighted sequence }\end{array}$

\section{Introduction}

Several studies using pathologic [1-4] and radiological [5-11] measures suggest that T1-hypointense lesions in multiple sclerosis (MS) ("black holes") are associated with more severe myelin and axonal loss. There is evidence for cross-sectional and longitudinal correlations of T1-hypointense-lesion load with clinical disability [10, 12-21].

The majority of new active MS lesions (65-80\%) [22] initially appear as hypointense on the unenhanced T1-weighted image (acute black holes, $\mathrm{ABH}$ ), probably because of edema, as well as early de- and remyelination. When the edema resolves and repair mechanisms take place, the T1-signal may gradually return to isointense. However, up to $40 \%$ [23] of ABH remain hypointense over time (persistent black holes, PBH). Published post hoc analyses of phase II trials suggested that this proportion is lower with some established [24, 25] and experimental agents [26, 27].

BHT-3009 is an experimental treatment for MS. It is a DNA vaccine that encodes full-length human myelin basic protein (MBP) and might induce antigen-specific tolerance. In a placebo-controlled phase II trial with 289 RRMS patients, a reduction of disease activity, assessed by MRI measures, was shown for low-dose $(0.5 \mathrm{mg})$ BHT-3009 [28]. Given BHT-3009's proposed mechanism of action, it may be most effective in patients with strong pre-treatment anti-MBP immune responses. Indeed, higher levels of pre- treatment anti-myelin basic protein (anti-MBP) antibodies in the cerebrospinal fluid (CSF) as well as in serum correlated with a reduction in MRI activity [28] and a reduction in MRI and clinical activity (relapse rate) [29], respectively.

Using the MRI data from the phase II trial, we aimed to determine the effect of BHT-3009 on the evolution of new inflammatory lesions (candidate lesions, CL) into $\mathrm{PBH}$. We hypothesized that treatment with BHT-3009 reduces the risk of developing PBH compared to placebo. We then performed an exploratory analysis to examine potential treatment effects (1) in different lesions subgroups (gadolinium-enhancing $\mathrm{CL}$ and $\mathrm{ABH}$ ) and (2) regarding different types of PBH (reduction of risk of large and strongly hypointense $\mathrm{PBH}$ ).

A second objective of this study was to examine whether a potential effect of BHT-3009 on the evolution of new lesions to persistent black holes changes with pre-treatment anti-MBP antibody levels. For this objective, we used the antibody levels in serum and hypothesized that patients with higher antibody levels would benefit more from treatment with BHT-3009.

\section{Methods}

\section{Patient population}

In the placebo-controlled, double-blind, multicenter phase II trial with BHT-3009 [28], 289 RRMS patients were randomly assigned 1:1:1 to receive intramuscular injections of either $0.5 \mathrm{mg}$ BHT-3009, $1.5 \mathrm{mg}$ BHT-3009, or placebo. The study has been performed in accordance with the ethical standards laid down in the 1964 Declaration of Helsinki and approved from the local ethical standards committee. All persons gave their informed consent prior to their inclusion in the phase II study. BHT-3009 was administered every 2 weeks for the first 4 weeks, then every 4 weeks thereafter for a total of 13 doses ( 44 weeks). Inclusion and exclusion criteria have been fully described elsewhere [28]. We performed a blinded-to-treatment assignment analysis of 157 out of the 289 patients, who had $\geq 1$ new T2-lesion at week 8 and/or week 16 (CL). Two patients with a total of $13 \mathrm{CL}$ were excluded because of insufficient MRI data (final $n=155$ ). The clinical, demographic, and imaging characteristics of the 155 patients included in this analysis are presented in Table 1.

Blood from 87 patients participating in the phase II study had been collected at week 0 and the levels of antiMBP IgM antibodies were measured in serum using peptide microarrays. The number of human MBP peptide epitopes recognized by serum antibodies (NUM) was calculated for each subject [29]. 
Table 1 Baseline characteristics of the 155 patients included in this study (patients with at least one CL at week 8 or 16)
EDSS Expanded Disability

Status Scale, $C L$ candidate lesions, $G d$ gadolinium, $A B H$ acute black holes

Note that age, disease duration, number of relapses in the previous 2 years, EDSS, T2and T1-hypointense lesion load were assessed at week 0 of the phase II study, whereas the number of candidate lesions (CL), acute black holes (ABH) and Gd-enhancing CLs per patient refer to week 8 and 16 of the study (time-point of the detection of CLs)

\begin{tabular}{|c|c|c|c|c|}
\hline Characteristics & $\begin{array}{l}\text { Placebo } \\
n=50\end{array}$ & $\begin{array}{l}0.5 \mathrm{mg} \text { BHT- } 3009 \\
n=57\end{array}$ & $\begin{array}{l}1.5 \mathrm{mg} \text { BНT-3009 } \\
n=48\end{array}$ & $p$ \\
\hline Gender (F/M) & $38 / 12$ & $41 / 16$ & $31 / 17$ & 0.5 \\
\hline \multicolumn{5}{|l|}{ Age (years) } \\
\hline Mean & 36.68 & 33.39 & 35.38 & \multirow[t]{3}{*}{0.2} \\
\hline Median & 37.5 & 33 & 35.5 & \\
\hline Range & $19-54$ & $18-54$ & $18-54$ & \\
\hline \multicolumn{5}{|c|}{ Disease duration (years) } \\
\hline Mean & 6.11 & 5.05 & 5.16 & \multirow[t]{3}{*}{0.5} \\
\hline Median & 5.13 & 3.37 & 3.47 & \\
\hline Range & $0.50-20.23$ & $0.32-21.50$ & $0.32-25.16$ & \\
\hline \multicolumn{5}{|c|}{ Number of relapses in previous 2 years } \\
\hline Mean & 1.92 & 2 & 2.19 & \multirow[t]{3}{*}{0.3} \\
\hline Median & 2 & 2 & 2 & \\
\hline Range & $1-4$ & $1-4$ & $1-8$ & \\
\hline \multicolumn{5}{|l|}{ EDSS } \\
\hline Mean & 2.44 & 2.39 & 2.32 & \multirow[t]{3}{*}{0.8} \\
\hline Median & 2.25 & 2.5 & 2 & \\
\hline Range & $1-3.5$ & $0-4$ & $1-3.5$ & \\
\hline \multicolumn{5}{|c|}{$\mathrm{T} 2$ lesion volume } \\
\hline Mean & 8,640 & 7,502 & 7,253 & \multirow[t]{3}{*}{0.7} \\
\hline Median & 5,853 & 5,130 & 4,239 & \\
\hline Range & $155-45,417$ & $223-44,135$ & $272-54,041$ & \\
\hline \multicolumn{5}{|c|}{ T1-hypointense lesion volume } \\
\hline Mean & 2,663 & 2,260 & 1,627 & \multirow[t]{3}{*}{0.2} \\
\hline Median & 1,004 & 979 & 690 & \\
\hline Range & $0-12,432$ & $0-15,002$ & $0-10,378$ & \\
\hline \multicolumn{5}{|l|}{ CL/patient } \\
\hline Mean & 3.6 & 4.19 & 4.81 & \multirow[t]{3}{*}{0.4} \\
\hline Median & 3 & 3 & 2 & \\
\hline Range & $1-13$ & $1-24$ & $1-20$ & \\
\hline \multicolumn{5}{|c|}{ Gd enhancing CL/patient } \\
\hline Mean & 2.46 & 3 & 3.5 & \multirow[t]{3}{*}{0.4} \\
\hline Median & 1 & 2 & 1.5 & \\
\hline Range & $0-9$ & $0-23$ & $0-14$ & \\
\hline \multicolumn{5}{|l|}{$\mathrm{ABH} /$ patient } \\
\hline Mean & 1.98 & 2.46 & 2.67 & \multirow[t]{3}{*}{0.5} \\
\hline Median & 1 & 1 & 1 & \\
\hline Range & $0-9$ & $0-15$ & $0-13$ & \\
\hline
\end{tabular}

The subgroup of patients with $\geq 1 \mathrm{CL}$ and available serum anti-MBP IgM levels $(n=46)$ was used to investigate the combined effects of BHT-3009 treatment and pre-treatment antibody levels on the evolution of $\mathrm{PBH}$.

\section{MRI acquisition protocol}

All patients underwent MR imaging of the brain at screening, week 8 , week 16, and monthly between week 28 and 48, according to a standardized protocol using a 1.5-Tesla magnet and applying the following sequences: proton density-weighted (PD-w: repetition time, TR: 2,800-3,800, echo time, TE: 14-40), T2-w conventional spin echo (TR: 2,800-3,800, TE: 80-120) and T1-w sequences (spin echo sequences, TR: 500-650, TE: 10-20), before and 5-10 min after the administration of gadolinium diethylenetriamine pentaacetate (Gd-DTPA $0.1 \mathrm{mmol} / \mathrm{kg}$ BW over $2 \mathrm{~min}$ ). For all sequences, axial oblique contiguous 3-mm 
slices were acquired with a matrix of $256 \times 256$ and field of view of 250 .

MRI analysis

MR images from all sites were gathered in the Medical Image Analysis Centre (MIAC, Basel University Hospital, Basel, Switzerland) and underwent quality control from qualified personnel. MR images of patients with $\geq 1$ candidate lesion were re-analyzed by one experienced rater (AP), blinded to treatment assignment. CL were defined as new lesions on MRI scans at week 8, compared with the screening MRI (week 0) and at week 16, compared with week 8; the presence and type of gadolinium (Gd) enhancement of CL was also recorded. CL were determined at weeks 8 and 16 and not at week 0 , to assure that they were truly new lesions and to allow treatment to exert its effect. CL were further tracked at week 16 (those from week 8), week 28 and 48 and the lesions' signal intensity on the T1-w pre-contrast SE, compared to surrounding normal appearing white matter (NAWM) was recorded (T1-isointense: signal intensity similar to NAWM and T1-hypointense: signal intensity lower than NAWM). T1-hypointense lesions should be sharply demarcated; diffuse, very mildly hypointense areas and areas with fuzzy borders were considered T1-isointense. T1-hypointense lesions at baseline (time-point of first detection of CL, i.e., week 8 or 16) were considered $\mathrm{ABH}$ and $\mathrm{T} 1$-hypointense lesions at week 48 were considered $\mathrm{PBH}$; $\mathrm{PBH}$ were small, if their longest diameter was $<5 \mathrm{~mm}$ and large, if their longest diameter was $\geq 5 \mathrm{~mm}$; they were strongly hypointense, if their signal intensity was similar to the CSF and mildly hypointense, if it was similar to normal appearing grey matter.

A repeat evaluation of T1-signal intensity at week 0 and week 48 in a sample of 50 lesions was performed by the same rater (AP), 6 months after the first evaluation. The intra-rater agreement for $\mathrm{ABH}$ and $\mathrm{PBH}$ was $96 \%$, with an intra-class correlation coefficient (ICC) value of $0.90(95 \%$ CI 0.85-0.93).

Statistics

Demographic, clinical, and MRI characteristics at week 0 and MRI characteristics at week 8 or 16 were compared between the three study arms using one-way ANOVA for continuous variables and a proportion test for the categorical variable gender.

We used generalized estimating equation (GEE) models with binomial error distribution to analyze the binary endpoint "incidence of a PBH" at week 48 for all CL being tracked. GEE models allow accounting for the fact that lesions are clustered within patients. Treatment with BHT3009 was used as three-level explanatory factor.

The same type of model was used for the exploratory analyses, i.e. analyzing the subgroups of $\mathrm{Gd}$ enhancing $\mathrm{CL}$ only ( $n=462)$ and ABH only $(n=367)$ and analyzing the incidence of large $\mathrm{PBH}$ and of strongly hypointense $\mathrm{PBH}$ for all CL.

To investigate the combined effects of pre-treatment antiMBP immune responsiveness and treatment with BHT3009 , the natural logarithm of NUM (number of detected MBP peptides), hereafter referred to as "log (antibodies)", and the interaction: "log (antibodies) $\times$ BHT-3009 treatment" were used as additional terms in the model.

\section{Results}

A total of $650 \mathrm{CL}$ were found in 155 patients of the phase II study. The total number of CL, and the proportions of $\mathrm{Gd}$ enhancing $\mathrm{CL}, \mathrm{ABH}$, and $\mathrm{PBH}$ in each treatment group are shown in Table 2.

We observed that the initial T1-signal-intensity of $\mathrm{CL}$ (ABH or not at week 8 or 16) was highly predictive of the outcome at week 48: the probability for a lesion to be $\mathrm{PBH}$

Table 2 Total number of candidate lesions (CL) as well as numbers and proportions of gadolinium (Gd)-enhancing CL, acute black holes $(\mathrm{ABH})$, and persistent black holes $(\mathrm{PBH})$ in the three treatment groups

\begin{tabular}{llll}
\hline & Placebo & 0.5 mg BHT-3009 & 1.5 mg BHT-3009 \\
\hline 650 CL (patients' $n=155)$ & 180 (patients' $n=50$ ) & 239 (patients' $n=57$ ) & 231 (patients' $n=48$ ) \\
Gd-enhancing CL & 123 & 171 & 168 \\
Gd-enhancing CL/CL (\%) & 68 & 72 & 72 \\
ABH & 99 & 140 & 128 \\
ABH/CL $(\%)$ & 55 & 59 & 55 \\
PBH & 45 & 48 & 45 \\
PBH/CL $(\%)$ & 25 & 20 & 19 \\
PBH/ABH $(\%)$ & 45 & 34 & 35 \\
\hline
\end{tabular}

Note that ABH were the candidate lesions that appeared as T1-hypointense at baseline (time-point of their first detection, i.e., week 8 or 16) 


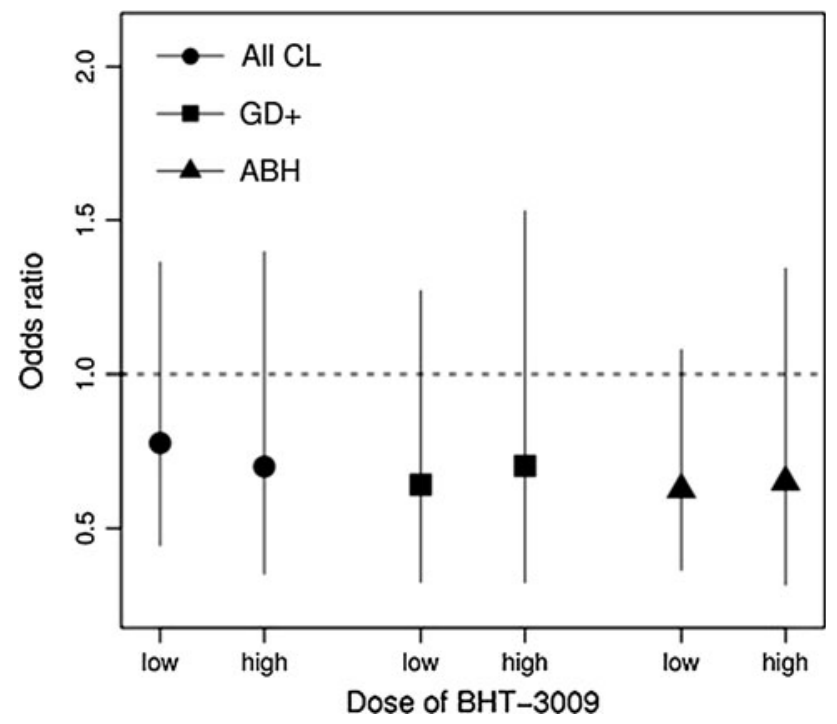

Fig. 1 Odds-ratio estimates with $95 \%$ CI for the effects of low$(0.5 \mathrm{mg})$ and high-dose $(1.5 \mathrm{mg})$ BHT-3009 versus placebo on the incidence of $\mathrm{PBH}$. A $95 \% \mathrm{CI}$ of the odds ratio including 1 corresponds to a non-significant difference. Odds ratios were calculated by GEE models including all $\mathrm{CL}$ or the subgroups of gadolinium-enhancing $\mathrm{CL}(\mathrm{GD}+)$ and acute black holes $(\mathrm{ABH})$ only

Table 3 Chi-square tests for the effects of treatment with BHT-3009, level of pre-treatment serum anti-MBP antibodies, and the interaction between the two on the incidence of persistent black holes (PBH)

\begin{tabular}{llll}
\hline & $d f$ & $\chi^{2}$ & $p$ \\
\hline Analysis including all CL & & & \\
$\quad$ Treatment with BHT-3009 & 2 & 0.85 & 0.65 \\
Log (antibodies) & 1 & 0.49 & 0.49 \\
$\quad$ Treatment with BHT-3009 $\times$ Log & 2 & 14.36 & 0.0008 \\
$\quad$ (antibodies) & & & \\
Analysis in the subgroup of ABH & & & \\
$\quad$ Treatment with BHT-3009 & 2 & 0.57 & 0.75 \\
$\quad$ Log (antibodies) & 1 & 0.2 & 0.65 \\
$\quad$ Treatment with BHT-3009 $\times$ Log & 2 & 8.27 & 0.016 \\
$\quad$ (antibodies) & & & \\
\hline
\end{tabular}

$M B P$ myelin basic protein, $C L$ candidate lesions, $A B H$ acute black holes $d f$ degrees of freedom

Chi-square tests are derived from "Analysis of Wald statistic tables" (GEE analogue to the ANOVA table)

at week 48 was much higher if it had been an $\mathrm{ABH}$ at baseline (odds ratio $=10.2, p<0.001$ ).

No effect of treatment with BHT-3009 was found on the evolution of $\mathrm{CL}$ to $\mathrm{PBH}$. Odds-ratio estimates for the effects of low- $(0.5 \mathrm{mg})$ and high-dose $(1.5 \mathrm{mg})$ BHT-3009 treatment versus placebo on the risk of $\mathrm{PBH}$ are shown in Fig. 1. Although odds-ratio estimates for both BHT-3009 treatment groups were below 1, no significant difference could be shown even when both active treatment groups

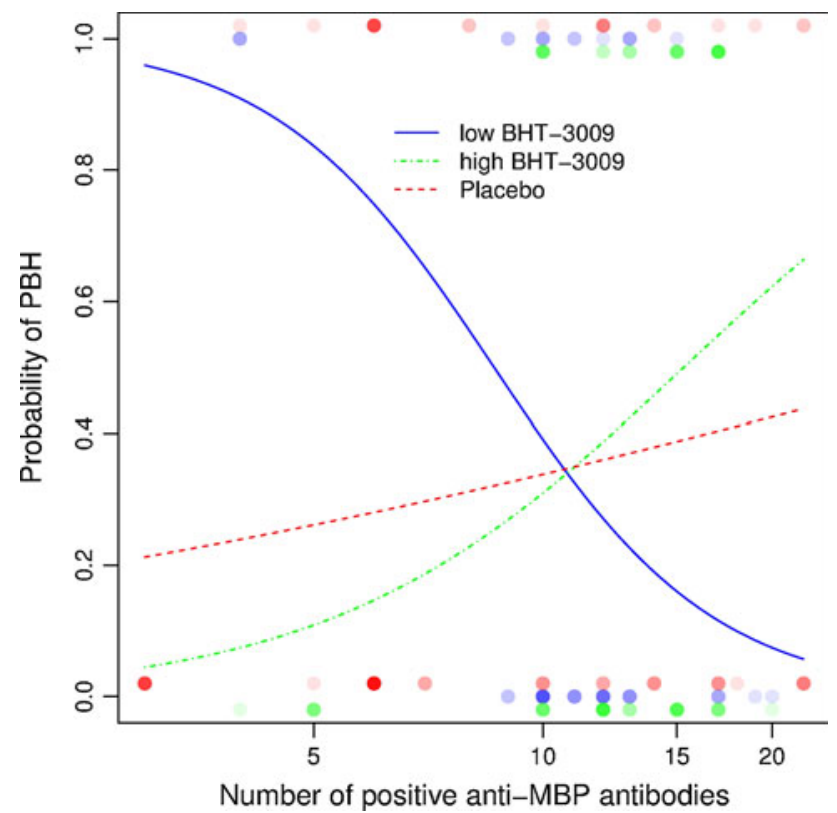

Fig. 2 Association between the probability of a CL developing into a $\mathrm{PBH}$ and the pre-treatment levels of anti-MBP antibodies (on a logarithmic scale) in all three treatment arms. The curves show the association between the probability of a CL developing into a $\mathrm{PBH}$ and the pre-treatment level of anti-MBP antibodies (on a logarithmic scale) by BHT-3009 treatment (indicated by color), as estimated by the logistic GEE model. The transparent, filled circles represent the observed CLs that either did or did not develop into $\mathrm{PBH}$, taking a value of 1 or 0 for the probability of $\mathrm{PBH}$, respectively. To improve legibility, the circles are jittered around 0 and 1 . This analysis included a total of 189 CLs from 46 patients with available anti-MBP antibody data. Darker circles represent multiple CLs. Note that the most effective treatment changes as we move along the range of antiMBP antibody levels, which is also reflected by the significant interaction between "treatment with BHT-3009" and "log (antibodies)" shown in Table 3

(high- and low-dose BHT-3009) together were compared with placebo. In line with this, our exploratory analysis neither showed a significant effect of BHT-3009-treatment on the risk of developing $\mathrm{PBH}$ in the subgroups of Gd-enhancing CL and ABH (Fig. 1), nor on the risk of developing large or strongly hypointense $\mathrm{PBH}$.

However, we found a significant interaction between treatment with BHT-3009 and the level of pre-treatment serum anti-MBP antibodies ["log (antibodies)"] (Table 3; Fig. 2). Patients receiving $0.5 \mathrm{mg}$ BHT-3009 showed a reduced risk of developing $\mathrm{PBH}$ with higher levels of antiMBP antibodies, compared to placebo $(p<0.01$ for the difference in slopes). On the contrary, patients receiving $1.5 \mathrm{mg}$ BHT-3009 showed a tendency for an increased risk of developing PBH with higher antibody levels, but the difference from placebo was not statistically significant. The outcome in patients receiving placebo was not affected by anti-MBPantibody levels. Similar results were found when this analysis was performed in the subgroup of ABH (Table 3). 
Finally, the 46 patients were divided into two subgroups based on the number of MBP peptides detected by IgM antibodies (NUM: $<12$ vs. $\geq 12$ ), to compare a potential treatment effect in the subgroup with higher antibody levels. This cut-off was chosen because it divides the data set into two groups of similar size and also roughly coincides with the intersection of lines in Fig. 2. However, we could not show a significant difference in the effect of BHT-3009 on the risk of PBH between these two patientsubgroups.

\section{Discussion}

In this post hoc analysis, we examined the evolution of new lesions to persistent black holes under treatment with BHT3009, an experimental DNA vaccine for MS patients, to investigate a potential treatment effect on promoting focal tissue repair. Although we observed in both BHT-3009 treated groups numerically lower proportions of $\mathrm{PBH} / \mathrm{CL}$ and $\mathrm{PBH} / \mathrm{ABH}$ (Table 2) and odds ratio estimates below 1 (Fig. 1), these differences were not statistically significant. Given the sample size, which included only a relatively small ( $n=155$ ) subgroup of the initially 289 randomized patients in the phase II study, it was not likely that we would be able to see statistically significant results. However, despite the small number of patients, we found a significant correlation between pre-treatment anti-MBP antibody responses and effect of BHT-3009 on the reduction of risk of $\mathrm{PBH}$. Most interestingly, the role of pretreatment serum antibody levels depended on the treatment group: in patients under placebo, antibody levels hardly affected the risk of $\mathrm{PBH}$; however, in patients on $0.5 \mathrm{mg}$ BHT-3009 anti-MBP antibodies seemed to play a positive role, since we observed a significant reduction in the risk of $\mathrm{PBH}$ compared with placebo, with increasing antibody levels. On the contrary, higher antibody levels tended to be associated with a higher risk to develop $\mathrm{PBH}$ in patients on $1.5 \mathrm{mg}$ BHT-3009 (however difference from placebo not statistically significant). These results indicate that pretreatment serum anti-MBP IgM levels could be used to identify populations of MS patients in which low-dose treatment with BHT-3009 might have a neuroprotective effect, promoting focal tissue repair. The lack of a significant interaction between BHT-3009 treatment and the binary variable $\geq 12$ versus $<12$ positive anti-MBP antibodies probably reflects the loss of statistical power that results from transforming the continuous predictor "log (antibodies)" into a binary predictor.

The interaction between reactivity against myelin basic protein and treatment effect of low-dose BHT-3009 supports the proposed mechanism of action of this DNA plasmid, as an immuno-tolerating treatment in MS. It is assumed that the pathogenesis of MS is largely due to antigen-specific autoimmunity with an essential role of myelin-specific $\mathrm{T}$ lymphocytes [30-32]. Our hypothesis is that after injection, low-dose BHT-3009 can produce myelin basic protein within antigen-presenting cells of the immune system and tolerize the MBP-reactive pathogenic T cells, thus attenuating the autoimmune disease process. Based on this, it is logical to assume that patients with higher pre-treatment immune responses against MBP would respond better to low-dose BHT-3009 treatment. Our findings support this hypothesis and are also consistent with the previously reported correlation between higher serum anti-MBP antibody levels and reduction of new lesions and relapses after 1 year of treatment with $0.5 \mathrm{mg}$ BHT-3009 [29].

The different results in the $1.5 \mathrm{mg}$ group are quite interesting. We observed a tendency for an increased risk of developing $\mathrm{PBH}$ in patients with higher antibody levels in this high dose group, as illustrated in Fig. 2. Thus, our findings confirm that the high dose of BHT-3009 is not effective in patients with higher pre-treatment immune responses against MBP. The fact that the $1.5-\mathrm{mg}$ dose of BHT-3009 is ineffective has been already suggested by the main results of the original phase II study, where a tendency for higher clinical and MRI activity as well as an increase in autoantibody reactivity in the CSF was observed in the 1.5-mg treatment arm [28]. This phenomenon of lack of efficacy or even deterioration with higher doses is also known from other antigen-specific therapies and might be due to an induction of a Th1, instead of Th2 immune response [33]. Our observation that this effect may be more pronounced in patients with stronger immune responses (in this case against MBP), makes sense. In these patients, the higher dose of antigen may have led to an excessive formation of MBP-antibody complexes in vivo, which result in overstimulation of the immune system and eventually to a more destructive inflammation in the central nervous system, reflected by more frequent formation of persistent black holes. Another possibility is that BHT-3009 in a dose of $1.5 \mathrm{mg}$ contains relatively high numbers of residual, consensus immunostimulatory cytosine-phosphate-guanine (CpG) motifs. These motifs could induce interferon- $\gamma$ production, causing an exacerbation of the disease and thus eliminating the tolerogenic effect of the plasmid [28].

Our results suggest that not only inflammatory activity but also focal tissue repair could depend on the relation of low-dose antigen-specific treatment and high pre-treatment immune response of the patients. This is important for two reasons: (1) it is compatible with the assumed mechanism of action of low-dose BHT-3009, at least in a specific population of MS patients and (2) it suggests an association between anti-inflammatory and neuroprotective treatment effects in MS. The latest is in line with other studies showing that immunomodulatory drugs reducing inflammatory 
activity can also reduce the risk of new persistent black holes [24, 25, 27, 34, 35].

It is interesting that the odds ratios of new lesions becoming $\mathrm{PBH}$ are significantly higher for lesions that initially appear as T1-hypointense. This emphasizes that although the initial T1-hypointensity of a new lesion often (approximately 60\%) [23] resolves over time, it is still a negative prognostic factor, which increases the risk of persistent hypointensity.

In conclusion, we could not show a significant treatment effect of BHT-3009 on the evolution of CL to PBH in general. However, an effect of low-dose $(0.5 \mathrm{mg})$ BHT3009 versus placebo in patients with high pre-treatment levels of anti-MBP antibodies in serum is suggested. Such dependence of treatment effect on the patients' pre-treatment immune responses needs further confirmation in prospective studies but-being consistent with an immunetolerating mechanism of action of BHT-3009_encourages further research with antigen-specific agents in MS.

Acknowledgments The original study was supported by Bayhill Therapeutics. A. Papadopoulou takes full responsibility for the data, the analyses and interpretation, and the conduct of the research, has full access to all of the data, and has the right to publish any and all data separate and apart from any sponsor. We acknowledge the support of Pascal Kuster (MIAC, Basel, Switzerland) and Marcus Weber (Neurology Clinic, Basel, Switzerland) in technical issues as well as the advice and guidance through this study of Danilo Marzetti, Dr. Nicole Müller-Lenke, Dr. Kerstin Bendfeldt (MIAC, Basel, Switzerland) and Thomas Fabbro, PhD (Clinical Trial Unit, University Hospital Basel, Switzerland).

Conflicts of interest The original study was supported by Bayhill Therapeutics. Athina Papadopoulou, Stefanie von Felten and Stefan Traud have no conflict of interest. Amena Rahman is paid as a consultant from Bayhill Therapeutics. Joanne Quan and Hideki Garren are employees of Bayhill Therapeutics and hold Bayhill stock options. Robert King is employed full-time by Bayhill Therapeutics and receives stock and financial compensation. Lawrence Steinman consults for Bayhill Therapeutics and serves on their Board of Directors. Gary Cutter has received Consulting, Speaking, \& Advisory Boards fees from Bayhill Therapeutics. Ludwig Kappos' institution has received payments for his participation as principal investigator, member or chair of planning and steering committees or advisory boards in corporate-sponsored clinical trials in multiple sclerosis and other neurological diseases. Sponsoring pharmaceutical companies for these trials include Bayhill Therapeutics. Ernst Wilhelm Radue has received research support (mainly for MS projects) from Bayhill Therapeutics.

\section{References}

1. Barkhof F, Brueck W, De Groot C et al (2003) Remyelinated lesions in multiple sclerosis magnetic resonance image appearance. Arch Neurol 60:1073-1081

2. Bitsch A, Kuhlmann T, Stadelmann C, Lassmann H, Lucchinetti C, Bruck W (2001) A longitudinal MRI study of histopathologically defined hypointense multiple sclerosis lesions. Ann Neurol 49:793-796
3. Brueck W, Bitsch A, Kolenda H, Brück Y, Stiefel M, Lassmann H (1997) Inflammatory central nervous system demyelination: correlation of magnetic resonance imaging findings with lesion pathology. Ann Neurol 42:783-793

4. van Walderveen MA, Kamphorst W, Scheltens P et al (1998) Histopathologic correlate of hypointense lesions on T1-weighted spin-echo MRI in multiple sclerosis. Neurology 50:1282-1288

5. Bitsch A, Bruhn H, Vougioukas V et al (1999) Inflammatory CNS demyelination: histopathologic correlation with in vivo quantitative proton MR spectroscopy. Am J Neuroradiol 20:1619-1627

6. Karampekios S, Papanikolaou N, Papadaki E et al (2005) Quantification of magnetization transfer rate and native $\mathrm{T} 1$ relaxation time of the brain: correlation with magnetization transfer ratio measurements in patients with multiple sclerosis. Neuroradiology 47:189-196

7. Levesque I, Sled JG, Narayanan S et al (2005) The role of edema and demyelination in chronic T1 black holes: a quantitative magnetization transfer study. J Magn Reson Imaging 21:103-110

8. Otaduy MC, Callegaro D, Bacheschi LA, Leite CC (2006) Correlation of magnetization transfer and diffusion magnetic resonance imaging in multiple sclerosis. Mult Scler 12:754-759

9. Rovira A, Alonso J, Cucurella G et al (1999) Evolution of multiple sclerosis lesions on serial contrast-enhanced T1-weighted and magnetization-transfer MR images. Am J Neuroradiol 20:1939-1945

10. van Waesberghe JH, van Buchem MA, Filippi M et al (1998) MR outcome parameters in multiple sclerosis: comparison of surfacebased thresholding segmentation and magnetization transfer ratio histographic analysis in relation to disability (a preliminary note). Am J Neuroradiol 19:1857-1862

11. van Walderveen MA, Barkhof F, Pouwels PJ, van Schijndel RA, Polman CH, Castelijns JA (1999) Neuronal damage in T1-hypointense multiple sclerosis lesions demonstrated in vivo using proton magnetic resonance spectroscopy. Ann Neurol 46:79-87

12. Iannucci G, Minicucci L, Rodegher M, Sormani MP, Comi G, Filippi M (1999) Correlations between clinical and MRI involvement in multiple sclerosis: assessment using $\mathrm{T}(1), \mathrm{T}(2)$ and MT histograms. J Neurol Sci 171:121-129

13. Minneboo A, Uitdehaag BM, Jongen P et al (2009) Association between MRI parameters and the MS severity scale: a 12 year follow-up study. Mult Scler 15:632-637

14. Nijeholt GJ, van Walderveen MA, Castelijns JA et al (1998) Brain and spinal cord abnormalities in multiple sclerosis. Correlation between MRI parameters, clinical subtypes and symptoms. Brain 121(Pt 4):687-697

15. Rovaris M, Comi G, Rocca MA et al (1999) Relevance of hypointense lesions on fast fluid-attenuated inversion recovery MR images as a marker of disease severity in cases of multiple sclerosis. Am J Neuroradiol 20:813-820

16. Sailer M, Losseff NA, Wang L, Gawne-Cain ML, Thompson AJ, Miller DH (2001) T1 lesion load and cerebral atrophy as a marker for clinical progression in patients with multiple sclerosis. A prospective 18 months follow-up study. Eur J Neurol 8:37-42

17. Truyen L, van Waesberghe JH, van Walderveen MA et al (1996) Accumulation of hypointense lesions ("black holes") on T1 spinecho MRI correlates with disease progression in multiple sclerosis. Neurology 47:1469-1476

18. van Walderveen MA, Barkhof F, Hommes OR et al (1995) Correlating MRI and clinical disease activity in multiple sclerosis: relevance of hypointense lesions on short-TR/short-TE (T1-weighted) spin-echo images. Neurology 45:1684-1690

19. van Walderveen MA, Truyen L, van Oosten BW et al (1999) Development of hypointense lesions on T1-weighted spin-echo magnetic resonance images in multiple sclerosis: relation to inflammatory activity. Arch Neurol 56:345-351 
20. van Walderveen MA, Lycklama ANG, Ader HJ et al (2001) Hypointense lesions on T1-weighted spin-echo magnetic resonance imaging: relation to clinical characteristics in subgroups of patients with multiple sclerosis. Arch Neurol 58:76-81

21. Zivadinov R, Leist TP (2005) Clinical-magnetic resonance imaging correlations in multiple sclerosis. J Neuroimaging $15: 10 \mathrm{~S}-21 \mathrm{~S}$

22. van Waesberghe $\mathrm{JH}$, van Walderveen MA, Castelijns JA et al (1998) Patterns of lesion development in multiple sclerosis: longitudinal observations with T1-weighted spin-echo and magnetization transfer MR. Am J Neuroradiol 19:675-683

23. Rovira A, Leon A (2008) MR in the diagnosis and monitoring of multiple sclerosis: an overview. Eur J Radiol 67:409-414

24. Dalton CM, Miszkiel KA, Barker GJ et al (2004) Effect of natalizumab on conversion of gadolinium enhancing lesions to $\mathrm{T} 1$ hypointense lesions in relapsing multiple sclerosis. J Neurol 251:407-413

25. Filippi M, Rovaris M, Rocca MA, Sormani MP, Wolinsky JS, Comi G (2001) Glatiramer acetate reduces the proportion of new MS lesions evolving into "black holes". Neurology 57:731-733

26. Barkhof F, Hulst HE, Drulovic J, Uitdehaag BM, Matsuda K, Landin R (2010) MN166-001 Investigators. Ibudilast in relapsing-remitting multiple sclerosis: a neuroprotectant? Neurology 74(13):1033-1040

27. MacManus DG, Miller D, Kappos L, et al (2008) The effect of BG00012 on conversion of gadolinium-enhancing lesions to T1-hypointense lesions. Mult Scler 14:163
28. Garren H, Robinson WH, Krasulova E et al (2008) Phase 2 trial of a DNA vaccine encoding myelin basic protein for multiple sclerosis. Ann Neurol 63:611-620

29. Garren H, Robinson WH, Rahman A, King R, Utz PJ, Steinman L (2010) Baseline plasma anti-MBP antibody levels correlate with subject response to BHT-3009, a novel, antigen-specific tolerizing DNA vaccine therapy for MS patients. Mult Scler p417

30. Frohman EM, Racke MK, Raine CS (2006) Multiple sclerosisthe plaque and its pathogenesis. N Engl J Med 354:942-955

31. Sospedra M, Martin R (2005) Immunology of multiple sclerosis. Annu Rev Immunol 23:683-747

32. Genain CP, Cannella B, Hauser SL, Raine CS (1999) Identification of autoantibodies associated with myelin damage in multiple sclerosis. Nat Med 5:170-175

33. Bielekova B, Goodwin B, Richert N et al (2000) Encephalitogenic potential of the myelin basic protein peptide (amino acids 83-99) in multiple sclerosis: results of a phase II clinical trial with an altered peptide ligand. Nat Med 6:1167-1175

34. Cadavid D, Cheriyan J, Skurnick J, Lincoln JA, Wolansky LJ, Cook SD (2009) New acute and chronic black holes in patients with multiple sclerosis randomised to interferon beta- $1 \mathrm{~b}$ or glatiramer acetate. J Neurol Neurosurg Psychiatry 80:1337-1343

35. Dalton CM, Miszkiel KA, Barker GJ et al (2004) Effect of natalizumab on conversion of gadolinium enhancing lesions to T1 hypointense lesions in relapsing multiple sclerosis. J Neurol 251:407-413 